\title{
Bright Polarized Single-Photon Source Based on a Linear Dipole
}

\author{
S. E. Thomas $\odot,{ }^{1}$ M. Billard, ${ }^{2}$ N. Coste,,${ }^{1,2}$ S. C. Wein, ${ }^{3}$ Priya $\odot,{ }^{1}$ H. Ollivier, ${ }^{1}$ O. Krebs $\odot,{ }^{1}$ \\ L. Tazaïrt, ${ }^{1}$ A. Harouri, ${ }^{1}$ A. Lemaitre, ${ }^{1}$ I. Sagnes, ${ }^{1}$ C. Anton $\odot,{ }^{1}$ L. Lanco,,${ }^{1,4}$ N. Somaschi, ${ }^{2}$ \\ J. C. Loredo, ${ }^{1, *}$ and P. Senellart ${ }^{1, \dagger}$ \\ ${ }^{1}$ Centre for Nanosciences and Nanotechnology, CNRS, Universite Paris-Saclay, \\ UMR 9001, 10 Boulevard Thomas Gobert, 91120 Palaiseau, France \\ ${ }^{2}$ Quandela SAS, 10 Boulevard Thomas Gobert, 91120 Palaiseau, France \\ ${ }^{3}$ Institute for Quantum Science and Technology and Department of Physics and Astronomy, \\ University of Calgary, Calgary, Alberta T2N 1N4, Canada \\ ${ }^{4}$ Université de Paris, Centre for Nanoscience and Nanotechnology (C2N), F-91120 Palaiseau, France
}

(Received 14 December 2020; revised 15 March 2021; accepted 12 May 2021; published 11 June 2021; corrected 15 December 2021)

\begin{abstract}
Semiconductor quantum dots in cavities are promising single-photon sources. Here, we present a path to deterministic operation, by harnessing the intrinsic linear dipole in a neutral quantum dot via phonon-assisted excitation. This enables emission of fully polarized single photons, with a measured degree of linear polarization up to $0.994 \pm 0.007$, and high population inversion— $85 \%$ as high as resonant excitation. We demonstrate a single-photon source with a polarized first lens brightness of $0.50 \pm 0.01$, a single-photon purity of $0.954 \pm 0.001$, and single-photon indistinguishability of $0.909 \pm 0.004$.
\end{abstract}

DOI: 10.1103/PhysRevLett.126.233601

The path towards an optimal single-photon source requires finding a scheme in which single photons are generated in a well-defined spatial and polarization mode, with near-unity efficiency, purity, and indistinguishability. A device with these properties is highly sought after for the advancement of quantum technologies such as secure long-distance quantum communication [1] and quantum computing [2-4]. Several platforms towards optimized single-photon sources are being developed, including spontaneous parametric down-conversion (SPDC) [5-7] and four-wave mixing (FWM) [8,9]. These nonlinear optical sources have an intrinsic efficiency limitation, and various multiplexing schemes are currently being explored to overcome this [9-13]. Sources based on semiconductor quantum dots (QDs) in microcavities have recently shown their capability to deliver high purity, indistinguishable single photons with record brightness [14-18]. With an efficiency per photon more than 1 order of magnitude higher than sources based on frequency conversion, QD sources have already allowed a substantial scaling up of optical quantum computing [19].

Despite this impressive progress, current QD sources are still far from the ideal deterministic performance that would provide a single photon in a pure quantum state with unity

Published by the American Physical Society under the terms of the Creative Commons Attribution 4.0 International license. Further distribution of this work must maintain attribution to the author(s) and the published article's title, journal citation, and DOI. probability. This ambitious goal requires a scheme that maximizes every single parameter controlling the source efficiency, including full inversion of the quantum dot transition, followed by the emission of a single photon with unity quantum purity, and perfect collection of the photon into a well-defined polarized optical mode. Coherent control of a QD has allowed generation of single photons in pure quantum states [20], and near-unity population inversion has been obtained through more sophisticated techniques such as rapid adiabatic passage [21,22]. Unpolarized collection efficiencies of up to $78 \%$ have been demonstrated with micropillar cavities [14,15]. However, obtaining such high performance into a polarized mode remains challenging. So far, the most efficient sources have used resonant excitation of a charged exciton state, which inherently emits unpolarized photons. When using unpolarized cavities, only half of the single photons are collected through polarization filtering [14,15,23]. Very recently, polarized cavities were used to accelerate spontaneous emission into one linear polarization, providing nearly a factor of 2 gain in the source efficiency [18,24]. Here, we propose a different path towards deterministic operation-making use of the linearly polarized optical transitions of a neutral quantum dot, which arise due to its natural anisotropy, to directly generate and collect polarized single photons.

Neutral QDs have a three-level energy structure, as shown in Fig. 1(a) where two excitonic eigenstates, labeled $|X\rangle$ and $|Y\rangle$, are separated by the fine structure splitting, $\Delta_{\text {FSS }}$ [25]. The optical transition between the QD ground state $|g\rangle$ and one of the exciton states corresponds to a 
linearly polarized dipole. While optical quantum technologies require polarized single photons, direct use of such a linear dipole has not been considered so far. Indeed, the generation of single photons with near-unity indistinguishability has only been reached under resonant excitation, a technique which is not applicable to a single linear dipole since the single photons have the same wavelength and polarization as the excitation laser and cannot be easily separated.

To overcome this limitation, we propose the use of an off-resonant phonon-assisted excitation scheme, which uses a slightly spectrally detuned laser that can be easily separated from the single photons. This excitation scheme has recently been theoretically proposed to reach both high QD inversion probability and high quantum purity [26-28], and relies on a detuned strong optical pulse that dresses the ground and excited states of the optical transition. During the pulse duration, the system relaxes between the dressed states through the emission of longitudinal-acoustic (LA) phonons. A strong occupation of the excited state is obtained following an adiabatic undressing of the dressed states during the switch-off of the excitation pulse. After the first observation of such phonon-assisted excitation [29], further theoretical studies predicted this excitation scheme should also provide near-unity single-photon purity, indistinguishability, and occupation probability $[27,28]$. The combination of this phonon-assisted excitation scheme with the use of a QD linear dipole thus appears as a promising route towards deterministic polarized single photon sources.

We study LA-phonon-assisted excitation of a linear dipole in a neutral QD deterministically located at the center of an electrically connected micropillar cavity [Fig. 1(b)] [14]. We study two samples (A and B) with a quality factor of around 4000 (10 000), respectively. The cavities are almost circular and exhibit two linearly polarized cavity modes typically separated by $\approx 70 \mu \mathrm{eV}$ $(\approx 30 \mu \mathrm{eV})$, more than 4 times smaller than the cavity linewidth of $\approx 300 \mu \mathrm{eV}(\approx 150 \mu \mathrm{eV})$. These devices have previously been shown to provide reproducible, highperformance single-photon generation under strictly resonant excitation, where the excitation laser was separated from the emitted photons via cross polarization. This reduced the polarized collection efficiency by a factor of 2 , and the polarized first lens brightness $\mathcal{B}_{\mathrm{FL}}$ - the probability per pulse to collect a polarized single photon at the first objective lens above the micropillar cavity-was limited to at best $\mathcal{B}_{\mathrm{FL}} \approx 25 \%[14,23]$.

In the present work, we implement LA-phonon-assisted excitation and set the excitation laser to be blue-detuned from the QD transition by approximately $0.6 \mathrm{~nm}$, corresponding to a phonon energy of around $1 \mathrm{meV}$. The excitation pulse is derived from a 3 ps pulsed Ti-sapphire laser centered at around $924.2 \mathrm{~nm}$, which is shaped using a 4-f filtering system to obtain pulses with tunable temporal (a)
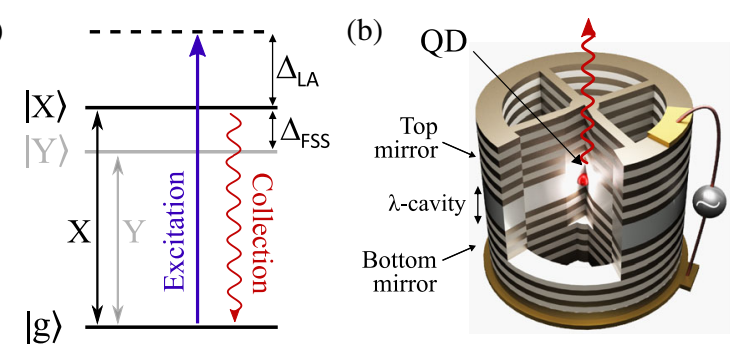

(c)

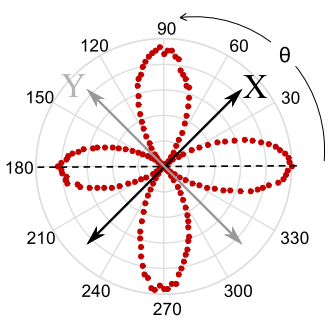

(d)

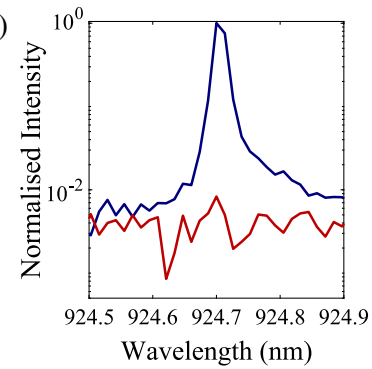

FIG. 1. (a) Energy level structure of a neutrally charged quantum dot, with exciton eigenstates $|X\rangle$ and $|Y\rangle$, separated in energy by $\Delta_{\mathrm{FSS}}$. The excitation pulse is blue-detuned from the transition by an energy of $\Delta_{\mathrm{LA}}$, or equivalently detuned in wavelength by $\Delta \lambda$. (b) A schematic of the quantum dot deterministically embedded at the center of an electrically contacted micropillar cavity [14] (c) Polar plot of the cross-polarized emission intensity measured under phonon-assisted excitation as a function of the incident polarization angle. (d) Spectrum of the emitted photons when exciting only the $X$ dipole of a neutral exciton and collecting in the parallel (blue) or orthogonal (red) basis.

length from 12 to 22 ps and negligible spectral overlap with the QD emission at $924.8 \mathrm{~nm}$. The single photon emission has a bandwidth of approximately $5 \mathrm{pm}(10 \mathrm{pm})$ for sample $A(B)$, and is separated from the pump laser using three high-transmission $0.8 \mathrm{~nm}$ bandpass spectral filters. The samples are placed in a closed-cycle cryostat and cooled to around $8 \mathrm{~K}(7 \mathrm{~K})$ for sample $A(B)$. For more experimental details, see the Supplemental Material [30].

We can visualize the two linear dipoles of the neutral QD by varying the linear polarization of the excitation laser, and measuring the single photon emission in the orthogonal polarization, as shown in Fig. 1(c). The intensity collected in cross polarization goes to zero when the polarization is aligned along one of the exciton linear dipoles and is maximum in between [23]. We can set the polarization of the excitation laser to excite just one of the excitonic dipoles, and the system reduces to an effective two-level system, $\{|g\rangle,|X\rangle\}$. The measured spectra of the single photons when collecting parallel or orthogonal to the $X$ polarization direction are shown in Fig. 1(d), evidencing strongly polarized emission. By calculating the integrated intensity measured in parallel (crossed) polarization $I_{\|}\left(I_{\perp}\right)$, we find a degree of linear polarization, $D_{\mathrm{LP}}=$ $\left[I_{\|}-I_{\perp}\right] /\left[I_{\|}+I_{\perp}\right]=0.994 \pm 0.007$ for device A1 on sample $A$. The same measurement was performed for 
two further different exciton-based devices on sample $A$ (devices A2 and A3) demonstrating linear polarization degree $D_{\mathrm{LP}}$ of $0.981 \pm 0.004$ and $0.971 \pm 0.001$. This polarization degree significantly exceeds what is accessible using the polarized Purcell effect in asymmetric cavities, with maximal values of 0.92 in optimized structures [18,24]—a limitation that arises from a compromise between reducing the cavity linewidth (to obtain large cavity splitting to linewidth ratios), and remaining in the weak coupling regime. With the current approach, there is no fundamental limit to the degree of polarization that could be reached. Even if the two linear dipoles present a slight nonorthogonality, which arises when the quantum dot shape and strain anisotropies do not coincide [32,33], it is always possible in principle to excite just a single dipole by aligning the excitation light to be orthogonal to the other dipole axis. The values of $D_{\mathrm{LP}}$ that we measured are slightly less than the ideal value of 1 due to a slight imperfection in the precise alignment of the polarization axis inside the cavity.

We now investigate the $\mathrm{QD}$ occupation probability, $p_{\mathrm{QD}}^{\mathrm{LA}}$, which can be obtained through LA-phonon-assisted excitation compared to the one achieved under resonant excitation $p_{\mathrm{QD}}^{\mathrm{RF}}$. To do so, we compare the single photon emission in a cross-polarization configuration, to be able to separate single photons from the laser for both excitation schemes. We study a charged exciton on sample $B$, which corresponds to a four-level system that acts as an effective two-level system when collecting the emission in cross polarization [23], in order to directly compare the two excitation schemes. Figure 2 presents the single-photon counts detected under strictly resonant excitation (black)

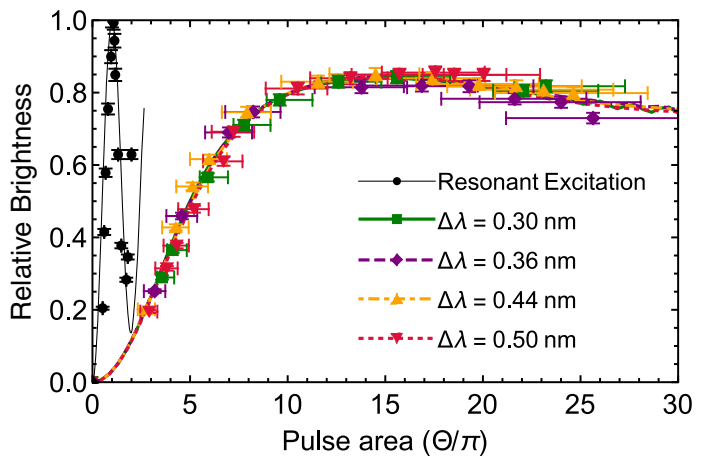

FIG. 2. Measured intensity of single-photon emission from a charged exciton on sample $B$ collected in cross polarization for resonant excitation (black) and LA-phonon-assisted excitation at various detunings, $\Delta \lambda$, as a function of the pulse area experienced by the quantum dot inside the cavity. The brightness is normalized to the maximum intensity achieved under resonant excitation. The sample is cooled to $7 \mathrm{~K}$ and the measured pulse duration is $(17 \pm 2) \mathrm{ps}$. The solid lines give the theoretical prediction based on the model in Ref. [28] for a quasi-Gaussian pulse with FWHM duration of $16 \mathrm{ps}$, and the lines are mostly superimposed for the detunings explored. and using LA-phonon-assisted excitation (colored) for a pulse duration of $(17 \pm 2)$ ps and various detunings from resonance as a function of the pulse area experienced by the quantum dot. The pulse area is proportional to the square root of the intracavity power, which is obtained by using the measured optical input power sent onto the cavity and the cavity transmission function at various detunings from the optical resonance. Higher input optical power is required to achieve the same intracavity pulse area for larger detunings. The error bars on the intracavity pulse area come from the uncertainty on the measured detunings. The resonant excitation data exhibits well-known Rabi oscillations reflecting the coherent control of the optical transition. Conversely, the LA-phonon-assisted excitation scheme does not show oscillations but a single rise and then slow decrease of the signal. The brightness of the singlephoton emission is much less sensitive to both the laser power and detuning for LA-phonon-assisted excitation as compared to resonant excitation, which shows that this scheme is significantly more robust to experimental drifts and instabilities. The solid lines in Fig. 2 show the theoretical predictions based on the model presented in Ref. [28] for our experimental parameters, assuming a quasi-Gaussian pulse with a FWHM duration of 16 ps (see Supplemental Material [30] for pulse shape). The theoretical model demonstrates good agreement with the experimental data. The measured occupation probability under LA-phonon-assisted excitation is as large as $0.85 \pm 0.01$ compared to that reached under resonant excitation, demonstrating the high efficacy of this scheme. Such high occupation probability is also observed on device A3 as shown below and is on par with previous observations [34]. Theory indicates that the QD occupation probability could be brought even closer to unity using a lower temperature and developing appropriate temporal shaping of the excitation laser [35].

We now focus on using a single linear dipole of a neutral exciton as a bright, polarized single-photon source. We investigate a neutral exciton (device A3) and measure the polarized brightness, single-photon purity, and indistinguishability of the emitted photons. Figure 3(a) shows the polarized first lens brightness and second-order autocorrelation, $g^{(2)}(0)$, as a function of the laser power for resonant and LA-phonon-assisted excitation. In order to evaluate the first lens brightness, $\mathcal{B}_{\mathrm{FL}}$, we precisely calibrate all losses and the detector efficiency of our experimental setup (see Supplemental Material [30] for the loss budget). For resonant excitation, the polarization of the excitation laser is aligned along one of the cavity axes and excites a combination of both $X$ and $Y$ optical transitions [23]. The brightness of the emission in cross polarization depends on the angle between the dipole axes and the cavity axes, which for this particular device (device 5 in Ref. [23]) leads to a $15 \%$ first lens brightness. For LA-phonon-assisted excitation the laser is aligned in polarization along the 
$X$ dipole axis, and blue-detuned by $0.85 \mathrm{~nm}$ with pulse duration of 13 ps. We can see that the polarized brightness reaches a value of $37 \%$ for these parameters-already more than a factor of 2 brighter than for resonant excitation.

Figure 3(b) shows the second-order autocorrelation $g^{(2)}(0)$ as a function of power for the two excitation schemes. At maximum brightness we measure very similar single-photon purity for LA-phonon-assisted excitation $\left[g^{(2)}(0)=0.030 \pm 0.002\right]$ and for resonant excitation $\left[g^{(2)}(0)=0.039 \pm 0.002\right]$. We further note that a slight reduction of the $g^{(2)}(0)$ is observed when increasing the excitation power for the phonon-assisted excitation scheme, in accordance with theoretical prediction [27] and in contrast to the case of resonant excitation. Preliminary theoretical study indicates that the minimal reachable $g^{(2)}(0)$ value strongly depends on the precise temporal shape of the laser and could be significantly reduced.

We also measure the Hong-Ou-Mandel interference visibility, $V_{\mathrm{HOM}}$, at maximum brightness for LA-phononassisted excitation. We measure $V_{\mathrm{HOM}}=0.851 \pm 0.002$, from which we extract the single-photon mean wave packet overlap [36] of $M_{\mathrm{s}}=\left[V_{\mathrm{HOM}}+g^{(2)}(0)\right] /\left[1-g^{(2)}(0)\right]=$ $0.911 \pm 0.003$. For resonant excitation we measured $M_{\mathrm{s}}=0.915 \pm 0.001$, demonstrating that the indistinguishability of the emitted photons under LA-phonon-assisted excitation is the same as the state-of-the-art values obtained under resonant excitation. We note that a high indistinguishability of around $84 \%$ had previously been demonstrated using phonon-assisted excitation of a QD in bulk [37]. Our study shows that the acceleration of spontaneous emission needed to obtain bright sources does not prevent high values of indistinguishability to be reached under phonon-assisted excitation. In fact the use of a cavity actually allows further improvement of the indistinguishability via suppression of the phonon sideband.
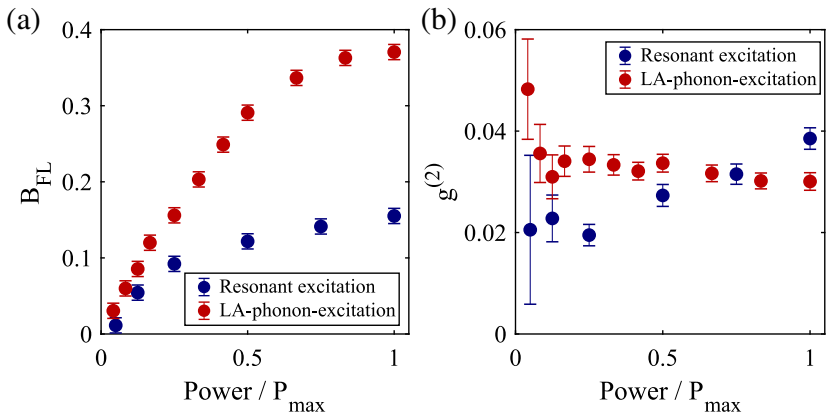

FIG. 3. (a) First lens brightness and (b) $g^{(2)}(0)$ as a function of the incident laser power for LA-phonon-assisted and resonant excitation, for device A3 on sample $A$. The power is scaled to the value required for maximum brightness for each excitation scheme, $P_{\max }$. The pulse duration is $13 \mathrm{ps}$ in both cases, and the LA-phonon-assisted laser is blue detuned from resonance by $0.85 \mathrm{~nm}$.
To find the optimal operational conditions for a bright single-photon source, we measure the single-photon purity, indistinguishability, and brightness of device A3 as a function of the detuning, $\Delta \lambda$, and pulse duration of the excitation laser, $\tau$. For each set of parameters, we adjust the excitation power to reach maximum brightness. The $g^{(2)}(0)$ remains mostly unchanged up to 18 ps pulse duration and increases for longer pulses due to a higher probability of reexcitation, as shown in Fig. 4(a). The single-photon indistinguishability is approximately constant at around 0.92 for all the explored parameters, as shown in Fig. 4(b). For one particular set of parameters we also measured the single-photon purity and indistinguishability with an additional $10 \mathrm{pm}$ bandwidth etalon filtering the collected photons, as shown by the open squares in Figs. 4(a)-4(b). For a detuning of $0.6 \mathrm{~nm}$ and a pulse duration of 20.5 ps we measured $g^{(2)}(0)=0.011 \pm 0.001$ and $M_{\mathrm{s}}=0.948 \pm 0.001$. This additional spectral filtering improves the $g^{(2)}(0)$ by suppressing any spurious laser photons as well as any extra spectrally broader photons due to reexcitation [36]. It also reduces the residual phonon-sideband emission and hence improves the indistinguishability. However, the etalon used in the present experimental implementation shows limited peak transmission. With improved, efficient spectral filtering it will be possible to reach significantly better values of singlephoton purity and indistinguishability while maintaining the high brightness.

Figure 4(c) shows that higher brightness is achieved for smaller detunings, which agrees with the theoretical prediction that the phonon-assisted excitation process becomes ineffective for larger detunings, and the optimal detuning will depend on the specific quantum dot and phonon coupling parameters [28]. The brightness also increases
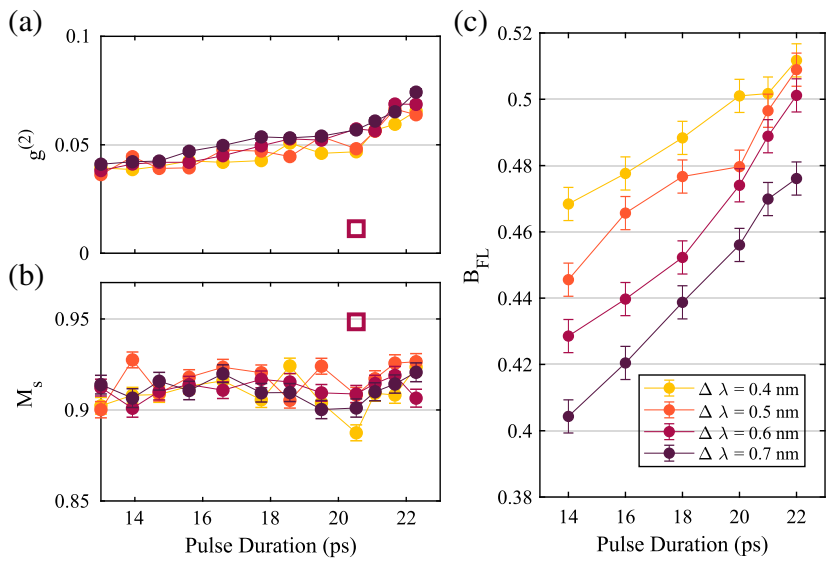

FIG. 4. (a) The second-order autocorrelation, $g^{(2)}(0)$, (b) single-photon indistinguishability $M_{\mathrm{s}}$, and (c) the first lens brightness, $\mathcal{B}_{\mathrm{FL}}$, as a function of the pulse duration and detuning $\Delta \lambda$ for device A3 on sample $A$. The open square points in (a) and (b) correspond to the values measured with an additional $10 \mathrm{pm}$ bandwidth etalon filtering the collected photons. 
for longer pulse durations, but with a slight degradation in the single-photon purity. For a detuning of $\Delta \lambda=0.4 \mathrm{~nm}$ and a pulse duration of $\tau=19.5 \mathrm{ps}$, we obtain a first-lens brightness of $B_{\mathrm{FL}}=0.50 \pm 0.01$, a single-photon purity of $\mathcal{P}=1-g^{(2)}(0)=0.954 \pm 0.001$ and a single-photon indistinguishability of $M_{\mathrm{s}}=0.909 \pm 0.004$. This is more than a factor of 3 higher than the brightness in resonant excitation for this device while maintaining state-of-the-art performance. Considering the extraction efficiency of our cavities $\eta_{\mathrm{ext}} \approx 0.65$, and the theoretically expected $p_{\mathrm{QD}}^{\mathrm{LA}} \approx 0.85$, the maximum expected first-lens brightness is $\mathcal{B}_{\mathrm{FL}}=\eta_{\mathrm{ext}} p_{\mathrm{QD}} \approx 0.55$, very close to our experimental observation. This value could be improved using cavities with higher extraction efficiencies of $\eta_{\text {ext }}=0.80$ [38], and by improving the QD occupation probability to near-unity via pulse shaping techniques [35]. In our current experimental implementation, the $50 \%$ first lens brightness corresponds to a detected count rate of $6 \mathrm{MHz}$ using a $69 \%$ efficient single photon detector and a laser repetition rate of $81 \mathrm{MHz}$. This value is limited by the low optical transmission of $17 \%$ of our experimental setup which could be considerably improved with optimized spectral filtering techniques such as custom fiber Bragg gratings which could filter the excitation laser from the single photons with an efficiency of over $90 \%$, and by direct coupling of the QD to an optical fiber with efficiencies of up to $90 \%$ [39].

In conclusion, we have reported on a new approach to bring QD-based single photon sources closer to deterministic operation by perfoming off-resonant phonon-assisted excitation of a linearly polarized dipole of a neutral QD. We demonstrated that phonon-assisted excitation can enable indistinguishabilities in the 90\%-95\% range, at the same level as resonant excitation, high occupation probability, and a significant increase in source brightness. As with resonant excitation, the indistinguishability reported here is limited by the moderate quality factor of our cavity. Near unity values should be reached using cavities with stronger Purcell effects as for resonant excitation [14], which is key for practical quantum technologies. The use of phononassisted excitation also enables a significant gain in stability and robustness, since the scheme is resilient to drifts in QD-laser detuning and excitation power. All these features are of great importance for practical applications in quantum technologies. Finally, we note that the use of phonon-assisted excitation in unpolarized cavities gives the possibility of exciting and collecting the photons in all polarization directions, which is critical for the generation of photonic cluster states using charged excitons [40], and this demonstration of indistinguishable single photons using this scheme is an important first step.

The authors would like to thank Andrew White and Marcelo Pereira de Almeida for experimental help. This work was partially supported by the ERC PoC PhoW, the IAD-ANR support ASTRID program Projet ANR-18ASTR-0024 LIGHT, the QuantERA ERA-NET Cofund in
Quantum Technologies, project HIPHOP, the EU Horizon 2020 FET OPEN project QLUSTER (Grant No. 862035), the EU Horizon 2020 FET OPEN project PHOQUSING (Grant No. 899544), the French RENATECH network, a public grant overseen by the French National Research Agency (ANR) as part of the "Investissements d'Avenir" programme (Labex Nano Saclay, reference: ANR-10LABX-0035). J. C. L. and C. A. acknowledge support from Marie SkłodowskaCurie Individual Fellowships SMUPHOS and SQUAPH, respectively. S. C. W. acknowledges support from NSERC (the Natural Sciences and Engineering Research Council), AITF (Alberta Innovates Technology Futures), and the SPIE Education Scholarships program. H. O. and N.C. acknowledge support from the Paris Ile-de-France Région in the framework of DIM SIRTEQ.

*juan.loredo1@gmail.com pascale.senellart-mardon@c2n.upsaclay.fr

[1] K. Takemoto et al., Quantum key distribution over $120 \mathrm{~km}$ using ultrahigh purity single-photon source and superconducting single-photon detectors, Sci. Rep. 5, 14383 (2015).

[2] S. Slussarenko and G. J. Pryde, Photonic quantum information processing: A concise review, Appl. Phys. Rev. 6, 041303 (2019).

[3] J. Wang, F. Sciarrino, A. Laing, and M. G. Thompson, Integrated photonic quantum technologies, Nat. Photonics 14, 273 (2020).

[4] T. Rudolph, Why I am optimistic about the silicon-photonic route to quantum computing, APL Photonics 2, 030901 (2017).

[5] S. Ramelow et al., Highly efficient heralding of entangled single photons, Opt. Express 21, 6707 (2013).

[6] M. M. Weston et al., Efficient and pure femtosecondpulse-length source of polarization-entangled photons, Opt. Express 24, 10869 (2016).

[7] F. Kaneda, K. Garay-Palmett, A. B. U'Ren, and P. G. Kwiat, Heralded single-photon source utilizing highly nondegenerate, spectrally factorable spontaneous parametric downconversion, Opt. Express 24, 10733 (2016).

[8] J. B. Spring et al., Chip-based array of near-identical, pure, heralded single-photon sources, Optica 4, 90 (2017).

[9] R. J. A. Francis-Jones, R. A. Hoggarth, and P. J. Mosley, All-fiber multiplexed source of high-purity single photons, Optica 3, 1270 (2016).

[10] C. Xiong et al., Active temporal multiplexing of indistinguishable heralded single photons, Nat. Commun. 7, 10853 (2016).

[11] C. Joshi, A. Farsi, S. Clemmen, S. Ramelow, and A. L. Gaeta, Frequency multiplexing for quasi-deterministic heralded single-photon sources, Nat. Commun. 9, 847 (2018).

[12] F. Kaneda and P. G. Kwiat, High-efficiency single-photon generation via large-scale active time multiplexing, Sci. Adv. 5, eaaw8586 (2019).

[13] E. Meyer-Scott, C. Silberhorn, and A. Migdall, Singlephoton sources: Approaching the ideal through multiplexing, Rev. Sci. Instrum. 91, 041101 (2020). 
[14] N. Somaschi et al., Near-optimal single-photon sources in the solid state, Nat. Photonics 10, 340 (2016).

[15] X. Ding et al., On-Demand Single Photons With High Extraction Efficiency and Near-Unity Indistinguishability from a Resonantly Driven Quantum Dot in a Micropillar, Phys. Rev. Lett. 116, 020401 (2016).

[16] P. Senellart, G. Solomon, and A. White, High-performance semiconductor quantum-dot single-photon sources, Nat. Nanotechnol. 12, 1026 (2017).

[17] Y.-M. He et al., Coherently driving a single quantum twolevel system with dichromatic laser pulses, Nat. Phys. 15, 941 (2019).

[18] H. Wang et al., Towards optimal single-photon sources from polarized microcavities, Nat. Photonics 13, 770 (2019).

[19] H. Wang et al., Boson Sampling with 20 Input Photons and a 60-mode Interferometer in a $10^{14}$-Dimensional Hilbert Space, Phys. Rev. Lett. 123, 250503 (2019).

[20] Y.-M. He et al., On-demand semiconductor single-photon source with near-unity indistinguishability, Nat. Nanotechnol. 8, 213 (2013).

[21] Y. Wu, I. M. Piper, M. Ediger, P. Brereton, E. R. Schmidgall, P. R. Eastham, M. Hugues, M. Hopkinson, and R. T. Phillips, Population Inversion in a Single InGaAs Quantum Dot Using the Method of Adiabatic Rapid Passage, Phys. Rev. Lett. 106, 067401 (2011).

[22] Y.-J.Wei et al., Deterministic and robust generation of single photons from a single quantum dot with $99.5 \%$ indistinguishability using adiabatic rapid passage, Nano Lett. 14, 6515 (2014).

[23] H. Ollivier et al., Reproducibility of high-performance quantum dot single-photon sources, ACS Photonics 7, 1050 (2020).

[24] N. Tomm et al., A bright and fast source of coherent single photons, Nat. Nanotechnol. 16, 399 (2021).

[25] M. Bayer et al., Fine structure of neutral and charged excitons in self-assembled in(ga)as/(al)gaas quantum dots, Phys. Rev. B 65, 195315 (2002).

[26] A. M. Barth, S. Lüker, A. Vagov, D. E. Reiter, T. Kuhn, and V. M. Axt, Fast and selective phonon-assisted state preparation of a quantum dot by adiabatic undressing, Phys. Rev. B 94, 045306 (2016).

[27] M. Cosacchi, F. Ungar, M. Cygorek, A. Vagov, and V. M. Axt, Emission-Frequency Separated High Quality SinglePhoton Sources Enabled by Phonons, Phys. Rev. Lett. 123, 017403 (2019).

[28] C. Gustin and S. Hughes, Efficient pulse-excitation techniques for single photon sources from quantum dots in optical cavities, Adv. Quantum Technol. 3, 1900073 (2020).

[29] J. H. Quilter, A. J. Brash, F. Liu, M. Glassl, A. M. Barth, V. M. Axt, A. J. Ramsay, M. S. Skolnick, and A. M. Fox, Phonon-Assisted Population Inversion of a Single
InGaAs/GaAs Quantum Dot by Pulsed Laser Excitation, Phys. Rev. Lett. 114, 137401 (2015).

[30] See Supplemental Material at http://link.aps.org/ supplemental/10.1103/PhysRevLett.126.233601 for further experimental details including a loss budget, and the temporal pulse shape of the laser used in the theoretical simulations, which includes Ref. [31]

[31] I. E. Zadeh, J. W. N. Los, R. B. M. Gourgues, V. Steinmetz, G. Bulgarini, S. M. Dobrovolskiy, V. Zwiller, and S. N. Dorenbos, Single-photon detectors combining high efficiency, high detection rates, and ultra-high timing resolution, APL Photonics 2, 111301 (2017).

[32] Y. Léger, L. Besombes, L. Maingault, and H. Mariette, Valence-band mixing in neutral, charged, and mn-doped self-assembled quantum dots, Phys. Rev. B 76, 045331 (2007).

[33] K. Kowalik, O. Krebs, A. Lemaître, J. A. Gaj, and P. Voisin, Optical alignment and polarization conversion of the neutral-exciton spin in individual InAs/GaAs quantum dots, Phys. Rev. B 77, 161305(R) (2008).

[34] P.-L. Ardelt et al., Dissipative preparation of the exciton and biexciton in self-assembled quantum dots on picosecond time scales, Phys. Rev. B 90, 241404(R) (2014).

[35] M. Glässl, A. M. Barth, and V. M. Axt, Proposed Robust and High-Fidelity Preparation of Excitons and Biexcitons in Semiconductor Quantum Dots Making Active Use of Phonons, Phys. Rev. Lett. 110, 147401 (2013).

[36] H. Ollivier et al., Hong-ou-mandel Interference with Imperfect Single Photon Sources, Phys. Rev. Lett. 126, 063602 (2021).

[37] M. Reindl, J. H. Weber, D. Huber, C. Schimpf, S. F. CovredaSilva, S. L. Portalupi, R. Trotta, P. Michler, and A. Rastelli, Highly indistinguishable single photons from incoherently excited quantum dots, Phys. Rev. B 100, 155420 (2019).

[38] O. Gazzano, S. Michaelis de Vasconcellos, C. Arnold, A. Nowak, E. Galopin, I. Sagnes, L. Lanco, A. Lemaître, and P. Senellart, Bright solid-state sources of indistinguishable single photons, Nat. Commun. 4, 1425 (2013).

[39] H. Snijders, J. A. Frey, J. Norman, V. P. Post, A. C. Gossard, J. E. Bowers, M. P. van Exter, W. Löffler, and D. Bouwmeester, Fiber-coupled cavity-QED source of identical single photons, Phys. Rev. Applied 9, 031002 (R) (2018).

[40] N. H. Lindner and T. Rudolph, Proposal for Pulsed OnDemand Sources of Photonic Cluster State Strings, Phys. Rev. Lett. 103, 113602 (2009).

Correction: Funding information in the second sentence in the Acknowledgements section was incomplete and has been fixed. 